\title{
Increasing Lignin Accumulation in Arabidopsis and Poplar by Overexpressing a CCoAOMT Gene from Dove Tree (Davidia involucrata Baill.)
}

Jian $\mathrm{Li}^{1,2}$, Xiaomin $\mathrm{Ji}^{1,2}$, Xujie Dong ${ }^{1,2}$, Fuxiang $\mathrm{Cao}^{2,3}$ and Meng $\mathrm{Li}^{1,2, *}$

1. College of Life Science and Technology, Central South University of Forestry and Technology, Changsha 410004, China

2. Hunan Research Center of Engineering Technology for Utilization of Environmental and Resources Plant, Changsha 410004, China

3. College of Horticulture and Landscape, Hunan Agricultural University, Changsha 410004, China

*Corresponding author: Meng Li (limeng0422@foxmail.com) 
Abstract: Rapid lignification occurring in the endocarp of dove tree results in the formation of a rigid and compact structure, which seriously hinders seed germination. A gene named DiCCoAOMT1, which encodes a hyperactive O-methyltransferase, was identified and thought to play a critical role in the process of endocarp lignification. In this study, the DiCCoAOMT1 gene was introduced into A. thaliana and poplar, respectively, to further verify its function. The lignin content was increased by $45 \%$ and $20 \%$ in the stems of transgenic A. thaliana and poplar lines, respectively. There was a positive correlation between the expression levels of DiCCoAOMT1 and lignin amount in transgenic lines. Furthermore, the shifts of lignin composition was indicated by the elevated $S / G$ ratio in transgenic poplar lines. Lignin accumulation was promoted specifically in the phloem cells, and the cells in secondary xylem was thickened in transgenic plants. In addition, lengthened pods and elevated plant height, and elongated petioles and internodes were observed in transgenic A. thaliana and poplar lines, respectively. Taken together, our data indicated that an endocarp-specific DiCCOAOMT1 gene could effectively increase lignin accumulation and alter lignin composition in both herbs and woody plants, which provides new insights to understand the regulatory mechanism of lignin biosynthesis and the biological significance of lignification in specific tissues.

Keywords: CCOAOMT; lignin; poplar (Populus tomentosa); Arabidopsis thaliana; dove tree (Davidia involucrata Baill.) 


\section{Introduction}

Lignin is one of the most important macromolecular organics in plants with multiple biological functions [1]. Lignin enhances the mechanical hardness and strength of plant body by filling the cellulose framework in cells. Besides the supporting function, lignin also play a role in defense against biotic and abiotic stress, such as avoiding biological invasion and water erosion, anti-bacterial, anti-oxidation, anti UV absorption and flame-retardant, which enhanced the adaptability of plants to diverse environment $[2,3]$. The lignin biosynthesis pathway has been extensively studied, and the genes and enzymes involved into this process have also been well identified. Lignin biosynthesis starts from the monolignols, which were derived from Phe, and the monolignols was converted into lignin monomer or units by a series of enzymes. The units resulting from the monolignols, when incorporated into the lignin polymer, are called guaiacyl (G), syringyl (S), and p-hydroxyphenyl (H) units [4]. During this process, PAL (phenylalanine ammonia-lyase), $\mathrm{C} 4 \mathrm{H}$ (cinnamate 4-hydroxylase), C3H (p-coumarate 3-hydroxylase), F5H (ferulate 5-hydroxylase), CAD (cinnamyl alcohol dehydrogenase), 4CL (4-coumarate: CoA ligase), CCR (cinnamoyl-CoA reductase), COMT (caffeic-acid-methyltransferase) and CCoAOMT (caffeoyl-CoA-methyltransferase) are considered as key enzymes, and their encoding genes were often used as targets of genetic modification for regulating lignin amount or alter the lignin composition in different species [5].

Lignin is widely distributed in plant cells, while mainly accumulated in the secondary wall of wood fiber cells. Wood fiber cells are concentrated in the xylem of plants, thus most related studies have been focused on the changes of lignin amount and composition in xylem. However, lignification is not limited just in xylem. In many woody plants, lignification can also be observed in roots, endocarps and pulps [5]. Different with that in xylem, the lignification process in these organs has to be completed in a limited time to form a specific structure, such as the cases in the shell of walnut or the endocarp of almond. The enzymes and genes involved into this process need to be tissue-specific and more efficient due to the spatiotemporal constrains. Therefore, the regulatory mechanism of lignin biosynthesis in fruit is 
supposed to be different with that in xylem. However, the studies regarding lignification in specific tissues is scarce.

Dove tree (Davidia involucrata Baill., Davidia hereafter) is a deciduous tree and the sole member of Davidiaceae. It is a relic species of Paleotropical flora in Tertiary period with many ancient and unique characteristics [6]. Davidia is an endangered species because its seed fertility is very low. The long-term seed dormancy of Davidia is believed to be one of the most important reasons for the low fecundity [7]. The seed dormancy of Davidia is determined by the unique structure of its endocarp. Rapid lignification occurring during the development process of Davidia endocarp, resulting in a thick, rigid and compact structure, which seriously hindered the seed respiration and material exchange $[8,9]$. In order to reveal the molecular mechanism of rapid lignification in endocarp, we performed transcriptome analysis and identified a gene named DiCCoAOMT1, which encodes a hyperactive O-methyltransferase. The expression of DiCCoAOMT1 is endocarp-specific, and its expression level was increasing along with the development of endocarp, thus DiCCoAOMT1 was speculated to play a critical role in regulating the lignification in Davidia endocarp [10].

CCoAOMT is an S-adenosyl-L-methionine (SAM) methyltransferase. It can transfer the methyl group on S-adenosylmethionine to the benzene ring carbon 3 position of coffee acyl-CoA, to form ferryl-CoA [4]. The CCOAOMT genes have been identified in Arabidopsis thaliana, rice (Oryza sativa), poplar (Populus tomentosa) and sorghum (Sorghum bicolor) etc., and were proved to be a key enzyme in lignin biosynthesis [11-14]. Inhibition of the expression level of CCOAOMT gene in tobacco (Nicotiana benthamiana) through expressing the antisense sequence of an $A h$ CCOAOMT gene from Acacia farnesiana resulted in the decrease of lignin content and the alternation of lignin components [15]. Similar results were also reported on the CCoAOMT genes from Pinus radiate [16] and Populus deltoids [17]. In maize (Zea mays), the lignin content decreased by $22.4 \%$ in the transgenic lines with reduced expression level of CCOAOMT gene through RNAi [18]. In Arabidopsis, the comt and ccoaomt mutants also showed significantly reduced lignin amount [14]. On the 
contrary, overexpression of the CCOAOMT gene PaCCoAOMT1 and PaCCoAOMT2 from Polypodiodes amoena resulted in increased lignin content in transgenic Arabidopsis [19]. While overexpression of a CcCCoAOMT1 gene from jute (Corchorus capsularis L.) [20], and a PmCCoAOMT gene from Japanese apricot (Prunus mume Sieb.) led to an increase of lignin amount in the reproductive organs of transgenic plants. These findings indicated that although all CCOAOMT genes encode similar enzymes, their tissue-specific expression result in different functions.

We have verified in vitro that DiCCoAOMT1 is a hyperactive O-methyltransferase, which might promote the lignification in Davidia endocarp. However, the expression of DiCCoAOMT1 is endocarp-specific, whether it can ectopically improve lignin biosynthesis is unknown. In this study, we introduced the DiCCoAOMT1 gene into Arabidopsis and poplar, respectively, by genetic transformation. Through overexpression in both herbs and woody plants, the gene function on lignin accumulation, lignin monomer composition, organ development and cell structure was further investigated. 


\section{Materials and Methods}

\section{1. Plant materials}

The seeds of Arabidopsis thaliana (L.) Heynh. ecotype Columbia-0 and the tissue culture seedlings of poplar (Populus tomentosa Carr.) are preserved in our lab. The tissue culture materials were grown at $22{ }^{\circ} \mathrm{C}$ under a $16 \mathrm{~h}$ light $/ 8 \mathrm{~h}$ dark photoperiod. The illumination was from cool-white fluorescent lights $(100-150 \mu \mathrm{mol}$ $\mathrm{m}^{-2} \mathrm{~s}^{-1}$ ). Wild type (WT) and transgenic Arabidopsis plants, and WT and transgenic poplar plants were grown in pots containing mixed medium (vermiculite: high-quality soil $=1: 3$ ). All plants were grown in controlled environment chambers at $20{ }^{\circ} \mathrm{C}$ under a $16 \mathrm{~h}$ light / $8 \mathrm{~h}$ dark photoperiod.

\section{2. DNA extraction, RNA extraction and cDNA synthesis}

The frozen samples were rapidly ground into powder in liquid nitrogen for nucleic acid extraction. The genome DNA was extracted by the CTAB method. The total RNA was extracted with an RNAPrep Pure Plant Kit (TianGen). RNA degradation and contamination were monitored on $1 \%$ agarose gels. RNA purity was checked with the NanoPhotometer spectrophotometer (IMPLEN). The first strand of cDNA was synthesised by reverse transcription with the Evo M-MLV reverse transcription kit (Takara). The cDNA was then diluted and used as a template for gene cloning and qPCR analysis, respectively.

\section{3. Genetic transformation in Arabidopsis and poplar}

The DNA fragment of target gene was inserted into the pRI101-AN vector by double digestion using BamHI and SalI and ligation using $\mathrm{T}_{4} \mathrm{DNA}$ ligase. Then the vector was transferred into Escherichia coli DH5 $\alpha$ for sequencing. The successfully constructed vector was transferred into Agrobacterium tumefaciens strain EHA105 for genetic transformation.

The floral dip method was used for genetic information of Arabidopsis [20]. Transformed Arabidopsis seeds were sowed on the MS medium [21] containing 50 $\mathrm{mg} / \mathrm{L}$ kanamycin and $200 \mathrm{mg} / \mathrm{L}$ cefotaxime and the seedlings with resistance to the antibiotics were selected. The homozygous transgenic lines were obtained by serial 
passage until no trait separation was observed in the progeny. More than 20 individual transgenic lines confirmed by PCR using gene-specific primers were generated, and the lines using for further study were selected according to the expression level of the target gene detected by qPCR.

The leaf disk method was used for genetic transformation of poplar [22]. The leaves using for transformation were collected from the 3-week-old tissue culture seedlings. Transformed poplar seedlings were transferred on the WPM medium [23] containing $25 \mathrm{mg} / \mathrm{L}$ kanamycin and $400 \mathrm{mg} / \mathrm{L}$ cefotaxime. The seedlings were grown under tissue culture conditions and the plants with resistance to the antibiotics were selected. More than 10 individual transgenic lines were generated, which were confirmed by PCR using gene-specific primers, and the lines using for further study were selected according to the expression level of the target gene detected by qPCR. The information of primers used in this study were listed in Table S1.

\section{4. Detecting gene expression levels by $q P C R$}

The cDNA samples of the leaves collected from 15-day-old Arabidopsis plants and the leaves collected from 60-day-old poplar plants were used as templates for qPCR analysis, respectively. An Arabidopsis gene, AtUBQ5 and a poplar gene, PtActin were used as reference genes for data normalisation. Real-time PCR was performed with the $2 \times$ SYBR Green Pro Taq HS Premix (Biotools, USA) and the ABI StepOne Plus-Type qPCR instrument (Applied Biosystems, USA). The $20.0 \mu \mathrm{L}$ qPCR reaction system contained $10.0 \mu \mathrm{L}$ of $2 \times$ SYBR Green Pro Taq HS Premix (Biotools, USA), 2.0 $\mu \mathrm{L}$ cDNA, $0.5 \mu \mathrm{L}$ of the forward primer $(10 \mu \mathrm{M}), 0.5 \mu \mathrm{L}$ of the reverse primer $(10 \mu \mathrm{M})$, $0.4 \mu \mathrm{L}$ of ROX Reference Dye (Biotools, USA) $(4 \mu \mathrm{M})$ and $6.5 \mu \mathrm{L}$ of RNase-free water. The PCR reaction program was as follows: predenaturation at $95{ }^{\circ} \mathrm{C}$ for $30 \mathrm{~s}$, then a cycle of denaturation at $95{ }^{\circ} \mathrm{C}$ for $15 \mathrm{~s}$ and annealing at $60{ }^{\circ} \mathrm{C}$ for $40 \mathrm{~s}$, repeated for 40 cycles. The relative expression levels were calculated according to the $2^{-\Delta \Delta \mathrm{Ct}}$ value [24]. Three independent biological replicates of each sample and three technical replicates of each biological replicate were used for qPCR analysis.

\section{5. Analysis of lignin content and lignin monomer composition}


Lignin content was detected using the acetyl bromide method described by Fukushima and Hatfield [25] with adjustments. The stems of 30-day-old Arabidopsis plants and 90-day-old poplar plants were collected for lignin content measuring. The lignin content was calculated according to the absorbance at $280 \mathrm{~nm}$. Three biological replicates of each sample were measured with technical repeats.

The lignin monomer composition was analysed using the method described by Lapierre et al. [26]. The stems were collected from 90-day-old transgenic and WT poplar plants. The stem samples were dried at $55^{\circ} \mathrm{C}$ for $24 \mathrm{~h}$, and then crushed by a crusher for measurement. For each sample, $10 \mathrm{mg}$ powder was measured by adding 10 $\mathrm{mL}$ of derivatization solution $(1 \mathrm{~mL}$ ethanethiol $+0.25 \mathrm{~mL}$ boron trifluoride etherate + $8.75 \mathrm{~mL}$ dioxane) and incubating at $100{ }^{\circ} \mathrm{C}$ for $1 \mathrm{~h}$. Then the reaction was terminated by cooling to room temperature, and the supernatant was diluted with $30 \mathrm{~mL}$ sterile water, and the $\mathrm{pH}$ was adjusted to 3.5 with $0.4 \mathrm{M} \mathrm{NaHCO}_{3}$. Extract 30 min with $30 \mathrm{~mL} \mathrm{CH}_{2} \mathrm{Cl}_{2}$, dry the organic phase with nitrogen, reconstitute n-hexane, and measure on an Agilent 7890B Meteorological Chromatography-Mass Spectrometer. The conditions were: inlet temperature $290{ }^{\circ} \mathrm{C}$, sample volume $1 \mu \mathrm{L}$, furnace temperature from $5{ }^{\circ} \mathrm{C}$ at $35^{\circ} \mathrm{C}$ $/ \min$ to $220^{\circ} \mathrm{C}$, and then at $0.5^{\circ} \mathrm{C} / \mathrm{min}$ to $230{ }^{\circ} \mathrm{C}$, then heated to $280{ }^{\circ} \mathrm{C}$ at $50{ }^{\circ} \mathrm{C} / \mathrm{min}$, and stayed for $7 \mathrm{~min}$. The ion source temperature is $280{ }^{\circ} \mathrm{C}$, the quadrupole temperature is $280{ }^{\circ} \mathrm{C}$, and the flow rate is $1 \mathrm{~mL} / \mathrm{min}$. The injection method is splitless, and the carrier gas is helium.

\section{6. Microscopic observation}

The paraffin sections of plant stems were prepared according to the method described by $\mathrm{He}$ et al. [27]. The stem segments were collected from 90-day-old transgenic poplar and Arabidopsis thaliana. Then the samples were fixed, dehydrated, transparent, dipped in wax, embedded, sliced, and patched to prepare paraffin sections. After the prepared paraffin sections were stained with $1 \%$ TBO (Toluidine Blue O) dye solution for 20-30 s, the dye solution was washed away, and the microscope (Leica DM2000 LED) was observed and photographed after mounting. After staining the prepared paraffin section with $5 \%$ resorcinol ethanol solution for 2-3 min, absorb 
the excess liquid with absorbent paper, then treat with $50 \%$ hydrochloric acid for 2-3 min, immediately observe and take a picture under the microscope.

\section{7. Statistical analysis}

One-way ANOVA was performed on the mean of the experimental results, and one-way analysis of variance was performed on the data by SPSS ver. 25.0 (SPSS Inc., USA) for Windows (SPSS Inc.) using with the honestly significant difference test of 1.s.d.LSD, Duncan's test (D), and Dunnett's test (E). The level of significance was set to $\mathrm{P}<0.05$. Pearson method was used for correlation analysis. 


\section{Results}

\section{1. Overexpression of DiCCoAOMT1 caused morphological change in transgenic} Arabidopsis

Transgenic Arabidopsis lines with relatively higher expression level of DiCCoAOMT1 were selected for further analysis. The expression level of DiCCoAOMT1 was 2291-, 358- and 1170-fold of that in wide type (WT) plants in A5, A7 and A16 lines, respectively (Figure 1e). The transgenic Arabidopsis plants exhibited a significant increase in vitality (Figure 1a). The plant height of transgenic lines was elevated by $12.70 \%$ on average compared with the WT plants (Figure 1b). In addition, significantly longer pods were observed in the transgenic plants (Figure 1c). The length of transgenic pods was 1.10 -fold on average of that of WT pods (Figure 1d). Correlation analysis indicated that there was a significant positive correlation between plant height and relative expression levels of DiCCoAOMT1 (Figure 1f).

\section{2. Lignin content increased in transgenic Arabidopsis}

Lignin content was compared between WT and transgenic Arabidopsis plants. The lignin content of different transgenic lines increased by $23.22 \%-43.85 \%$ compared to that of WT plants (Figure 2a). There was a positive correlation between the relative expression levels of DiCCoAOMT1 and lignin content, indicating that the high lignin content was determined by the overexpression of DiCCoAOMT1 (Figure $2 \mathrm{~b}$ ). Then histochemical staining in the cross sections of stems confirmed that lignin accumulation was increased in the xylem of transgenic plants (Figure 2c). Moreover, we observed that the stem diameter was obviously lengthened, and the cortex area was thickened in the transgenic lines. The size of vascular bundle cells increased, and the phloem fibers was lengthened, indicating a faster growth of primary phloem in the transgenic lines. Meanwhile, the proportion of pith area decreased, the size of pith cells significantly increased, whereas the number of pith cells decreased (Figure 2d). 


\section{3. Morphological change in transgenic poplar plants}

The DiCCoAOMT1 gene was then introduced into poplar to reveal its functions in woody plants. Three transgenic lines with different expression levels of DiCCoAOMT1 were selected for further analysis. The expression level of the target gene was 203-, 4202- and 2868-fold of that in WT plants in P3, P8 and P12 lines, respectively (Figure 3b). Different with the case in Arabidopsis, the plant height was not improved by overexpressing DiCCoAOMT1 in poplar. Instead, we noticed that the length of internode and petiole was obviously increased in the transgenic plants (Figure 3a). Significantly lengthened internodes and petioles were observed in the fifth true leaf of the transgenic poplar plants (Figure 3c and 3d). There was a positive correlation between internode length and relative expression level of DiCCoAOMT1, as well as between petiole length and relative expression level of DiCCoAOMT1 (Figure 3e).

\section{4. Lignin accumulation increased in transgenic poplar plants}

Lignin content was detected in the stems of transgenic poplar lines. The lignin content of P3, P8 and P12 lines increased by 11.47\%, 21.54\% and 13.63\% compared with that of WT plants, respectively (Figure 4a). There was a positive correlation between the relative expression level of DiCCoAOMT1 and lignin content (Figure 4b). Histochemical staining demonstrated that lignin accumulation was significantly increased in the primary and secondary xylem cells of transgenic plants (Figure 4c). Microscopic observation showed that the intrafascicular cambium and primary xylem were thickened, the pith rays were lengthened, and the pith cells were enlarged in the stems of transgenic lines. There was no obvious change in parenchyma and primary phloem cells of cortex.

\section{5. Lignin monomer composition was changed in transgenic polar plants}

In order to further reveal the function of DiCCoAOMT1, the lignin monomer composition in transgenic poplar stems was analyzed using GC-MS. The results 
showed that the G-unit content was significantly increased in P8 and P12, but not in P3. Similarly, the S-unit content was also increased in P8 and P12 but not in P3. Remarkably, the P8 line with highest expression level of DiCCoAOMT1 showed 7.32-fold and 8.68-fold of G-unit and S-unit content of that in WT plants, respectively. The H-type lignin monomer content was low in both WT and transgenic plants, while there was a slight increase in P3 and P8, but a decrease in P12 (Figure 5a). The S/G ratio was ranged from 0.78 to 1.03 in transgenic lines, which was significantly higher than that in WT plants (0.66), indicating that overexpression of DiCCoAOMT1 promoted the biosynthesis of S-unit more (Figure 5b). 


\section{Discussion}

The process of lignin biosynthesis is highly conserved in most plants, and its regulatory mechanism is complicated. As one of the key regulators, the CCoAOMT gene family has been a research hotspot for a long time. We have identified 14 CCOAOMT gene from the transcriptome data of Davidia. However, only one gene, DiCCoAOMT1, showed an endocarp-specific expression pattern. This finding indicated that fine division in gene family determines lignin biosynthesis in different tissues. Similar result were reported in other species. Among 7 CCOAOMT genes in A. thaliana, AtCCoAOMT1 was expressed in all tissues with the highest expression level. The expression levels of AtCCoAOMT4, AtCCoAOMT5 and AtCCoAOMT7 increased along with stem development. However, AtCCoAOMT2, AtCCoAOMT3 and AtCCOAOMT6 were only expressed in the later stages of stem development [28]. In the study in giant bamboo, the expression of DsCCoAOMT gene was up-regulated with the development of bamboo shoots, and maintained a high level in the metaphase of bamboo shoot development [29]. Therefore, although lignin biosynthesis in different tissues shares a same pathway, the selection of tissue-specific genes might be an important regulation mode to realize lignification at designated areas.

Endocarp is essential for the adaptations in seed protection and dispersal strategies in plants. Lignification in endocarp is a very different developmental program that requires unique sets of genes or enzymes [30]. The enzymatic activity of PAL, C4H, 4CL, CAD and POD increased rapidly in 30 days in the endocarps of peach (Prunus persica) [31]. Similar with our results, phenylpropanoid (PAL and $C 4 H$ ) and lignin (CCOAOMT, peroxidase and laccase) pathway genes were specifically induced in the endocarp layer over a 10 day time period. However, in walnut (Juglans regia), the enzymatic activity of both POD and PAL was decreased in endocarp during fruit development [32]. These evidences indicated that lignification in endocarps or fruits was under a very different control, which might be the reason for the lengthened pods we observed in transgenic Arabidopsis lines.

There was a positive correlation between expression levels of DiCCOAOMT1 and 
lignin content in both transgenic Arabidopsis and poplar lines (Fig. 2b and Fig. 4b), indicating the dosage effect of the function of DiCCoAOMT1. We observed that the cambium area was narrowed or replaced by the primary xylem cells in the stem cross section of transgenic poplar lines. However, this variation was not obvious in line P3 (Fig. 4c), in which the expression level of DiCCoAOMT1 was lowest. Similarly, the results of lignin monomer composition also shown that lignin monomer content was not altered in line P3 (Fig. 5a). These findings showed that DiCCoAOMT1 functioned in lignin biosynthesis under high dosage.

Consistent with our results, overexpression of a MICCOAOMT1 gene from Miscanthus lutarioriparius also resulted in thickened secondary cell wall in transgenic Arabidopsis [33]. However, different results have been found in the study of an LjCCoAOMT1 gene from Lonicera japonica. Overexpression of LjCCoAOMT1 gene did not cause variations in plant height and leaves in transgenic Rice (Oryza sativa L.) lines, but there were significant differences in the number of tillers and the number of grains per ear [34]. Although the transgenic lines of Arabidopsis and poplar both exhibited elevated vigor, they have different morphological variations (Fig. 1a and Fig. 3a). Higher lignin content might provide stronger mechanical support to the transgenic lines, meanwhile promote the growth rate of stems and leaves.

It is unexpected that overexpression of $\mathrm{DiCCoAOMT1}$ increased the $\mathrm{S} / \mathrm{G}$ rate in transgenic poplar lines. There are a number of reports proved that inhibiting CCOAOMT activity reduced the lignin content and G-unit content in transgenic plants, but the S-unit content was not affected, thus resulting in an increase of S/G ratio [17, 35, 36]. On the contrary, inhibition of COMT activity resulted in decrease of S-unit content, whereas G-unit content remains unchanged [37]. These evidences indicated that COMT is involved in the methylation of C5 position and specifically participates in the synthesis of S-unit, while CCoAOMT is mainly involved into the biosynthesis of G-unit. However, some studies found that CCOAOMT is not only involved in the synthesis of G-unit, but also in the synthesis of S-unit [38, 39]. DiCCoAOMT1 might promote S-unit biosynthesis through altering the conversion direction of conifer 
aldehyde [40]. Conifer aldehyde is located at the shunt node of G- and S-unit. It comes from two sources, including caffeic acid CoA and ferulyl CoA. We speculate that the high expression level of DiCCoAOMT1 caused massive accumulation of conifer aldehydes, and catalyzed the conversion from F5H to S-unit. In addition, overexpression of DiCCoAOMT1 also caused a slight increase of $\mathrm{H}$-unit content in some transgenic lines. Hydroxystilbenes, a new class of stilbenolignin polymers, were identified in the endocarp of some palm species, indicating that the lignin composition of endocarp is very different with those in other tissues. The endocarp of Davidia is much harder than that of its stems, indicating a different lignin composition in the endocarp. Whether the substrate of DiCCoAOMT1 is ferulyl CoA or other compounds remains to be further studied.

DiCCoAOMT1 was specifically expressed in endocarp, and its expression level was elevated along with the lignification process of endocarp. The upstream regulation of DiCCOAOMT1 is still a mystery. We cloned the promoter region of DiCCoAOMT1, and identified several cis-elements including a MBS (MYB binding site), a Sp1 element and a rbcS-CMA7a element involved in light response, and a TGACG-motif involved in the MeJA-responsiveness (unpublished data). This result indicated that the expression of DiCCoAOMT1 might be controlled by light and endogenous hormones, and transcription factors, such as MYB, could play a critical role in regulating CCOAOMT gene and lignin biosynthesis. This finding was also reported in the study in hawthorn (Crataegus spp.), which performed the comparing transcriptome analysis between soft-endocarp and hard-endocarp hawthorn, and identified some MYB and NAC transcription factors that could potentially control lignin and flavonoid biosynthesis specifically in endocarp [41]. MYB and NAC transcription factors were also reported to be closely related to lignin accumulation in loquat fruit $[42,43]$.

Taken together, we identified an endocarp-specific CCOAOMT gene and verified its function in both herb and woody plants. The target gene significantly increased lignin content and altered lignin composition in transgenic lines, indicating its critical 
role in regulating the rapid lignification and determining the long seed dormancy in Davidia.

\section{Conclusions}

In summary, in this study, we verified the function of a gene called DiCCOAOMT1 from the endocarp of Davidia in Arabidopsis and poplar. Overexpression of DiCCoAOMT1 led to organ enlargement in transgenic plants, which also showed enhanced vigor. Moreover, overexpression of DiCCoAOMT1 increased the lignin content by $45 \%$ and $20 \%$ in transgenic A. thaliana and poplar lines, respectively. Different with the function of CCOAOMT genes reported in other species, DiCCoAOMT1 contributed more on the biosynthesis of S-unit, thus increased the $\mathrm{S} / \mathrm{G}$ rate and altered the lignin composition in transgenic poplar lines. The spatiotemporal expression of DiCCoAOMT1 is important for the formation of the unique structure of Davidia endocarp, and the lignin composition of endocarp was supposed to be very different with that in xylem. Our results is not only laid a theoretical basis for elucidating the long seed dormancy of Davidia, but also important for understanding the molecular mechanism of lignification in specific organs. 


\section{Supplementary Materials:}

Figure S1: The content of G-, S- and H-unit of lignin monomer detected by GC-MS. a, WT; b, P3; c, P8; d, P12. Table S1: Information of primers applied in the present study.

Author Contributions: Meng Li and Fuxiang Cao designed the experimental scheme; Xujie Dong provided the endocarp materials and transcriptome data of D. involucrata; Jian Li and Xiaomin Ji performed experiments and data analysis; Meng Li and Jian Li wrote the manuscript. All authors have read and agreed to the published version of the manuscript.

Funding: This research was supported by grants from the Innovation Project of Forestry Science and Technology in Hunan Province (XLK201984) and the Key Research and Development Project of Hunan Province (2017NK2252).

Acknowledgements: The authority responsible for the D. involucrata resources is the Badagong Mountain Nature Reserve Management Division, who gave permission to collect the samples for our scientific research. We are grateful to the managers of Badagong Mountain Nature Reserve for their support of this study.

Conflicts of Interest: The authors declare no conflict of interest. 


\section{References}

1. Whetten, R. Lignin Biosynthesis. The Plant Cell 1995, 7, 1001-1013.

2. Baucher, M.; Halpin, C.; Petit-Conil, M. Boerjan, W. Lignin: Genetic Engineering and Impact on Pulping. Critical Reviews in Biochemistry and Molecular Biology 2003, 38, 305-350.

3. Vanholme, R.; Demedts, B.; Morreel, K.; Ralph, J.; Boerjan, W. Lignin

Biosynthesis and Structure. Plant Physiology 2010, 153, 895-905.

4. Boerjan, W.; Ralph, J.; Baucher. M. Lignin biosynthesis. Annual Review of Plant Biology 2003, 54, 519-546.

5. Simon, C.; Lion, C.; Huss, B.; Blervacq, A.S.; Spriet, C.; Guérardel, Y.; Biot, C.; Hawkins, S. BLISS: Shining a light on lignification in plants. Plant Signaling \& Behavior 2017, 12, e1359366.

6. Manchester, S.R. Leaves and fruits of Davidia (Cornales) from the Paleocene of North America. Systematic Botany 2002, 27, 368-382.

7. Chen, K.R. A preliminary report on the research of dormancy of Davidia involucrata seeds. Plant Physiology Communications 1988, 3, 24-28.

8. Wan, C.K. Anatomical study on seed dormancy of Dove tree(Davidia involucrata). Journal of Central South Forestry College 1988, 8, 35-39.

9. Li, M.; Dong, X.J.; Peng, J.Q.; Xu, W.; Ren, R.; Liu J.; Cao, F.X.; Liu, Z.M. De novo transcriptome sequencing and gene expression analysis reveal potential mechanisms of seed abortion in dove tree (Davidia involucrata Baill.). BMC Plant Biology 2016, 16, 82.

10. Wu, X.B.; Yan, Z.W.; Dong X.J.; Cao, F.X.; Peng, J.Q.; Li, M. Cloning and characterization of a CCOAOMT gene involved in rapid lignification of endocarp in dove tree (Davidia involucrata Baill.). Biotechnology \& Biotechnological Equipment 2019, 32, 1398-1406.

11. Wei, J.H.; Zhao, H.Y.; Zhang, J.Y.; Liu, H.R.; Song, Y.R. Cloning of cDNA Encoding CCoAOMT from Populus tomentosa and Down-regulation of Lignin Content in Transgenic Plant Expressing Antisense Gene. Acta Botanica Sinica 2001, 43, 
1179-1183.

12. Rakoczy, M.; Femiak, I.; Alejska, M.; Figlerowicz, M.; Podkowinski, J. Sorghum CCoAOMT and CCoAOMT-like gene evolution, structure, expression and the role of conserved amino acids in protein activity. Molecular Genetics and Genomics 2018, 293, 1-13.

13. Zhao, H.Y.; Sheng, Q.X.; Lü, S.Y. Characterization of three rice CCoAOMT genes. Chinese Science Bulletin 2004, 49, 1602-1606.

14. Do, C.T.; Pollet, B.; Thevenin, J.; Sibout, R.; Denoue, D.; Barriere, Y.; Lapierre, C.; Jouanin, L. Both caffeoyl Coenzyme A 3-0-methyltransferase 1 and caffeic acid 0-methyltransferase 1 are involved in redundant functions for lignin, flavonoids and sinapoyl malate biosynthesis in Arabidopsis. Planta 2007, 226, 1117-1129.

15. Pang, S.L.; Ong, S.S.; Lee, H.H.; Zamri, Z.; Kandasamy, K.I.; Choong, C.Y.; Wickneswari, R. Isolation and characterization of CCoAOMT in interspecific hybrid of Acacia auriculiformis x Acacia mangium --a key gene in lignin biosynthesis. Genetics and Molecular Research:GMR 2014, 13, 7217-7238.

16. Wagner, A.; Tobimatsu, Y.; Phillips, L.; Flint, H.; Torr, K.; Donaldson, L.; Pears, L.; Ralph, J. CCoAOMT suppression modifies lignin composition in Pinus radiata. The Plant Journal 2011, 67, 119-129.

17. Eom, I.Y.; Kim, K.H.; Lee, S.M.; Yi, Y.S.; Choi, J.W. Characterization of Chemical Composition in Poplar wood (Populus deltoides) by Suppression of CCoAOMT Gene Expression. Journal of the Korean Wood Science and Technology 2010, 38, 213-222.

18. Li, X.Y.; Chen, W.J.; Zhao, Y.; Xiang, Y.; Jiang, H.Y.; Zhu, S.W.; Cheng, B.J. Downregulation of caffeoyl-CoA O-methyltransferase (CCoAOMT) by RNA interference leads to reduced lignin production in maize straw. Genetics and Molecular Biology 2013, 36, 540-546.

19. Zhang, X.S.; Ni, R.; Wang, P.Y.; Zhu, T.T.; Sun, C.J.; Lou, H.X.; Cheng, A.X. Isolation and functional characterization of two Caffeoyl Coenzyme A 3-0-methyltransferases from the fern species Polypodiodes amoena. Plant Physiology and Biochemistry 2019, 136, 169-177. 
20. Zhang, G.Y.; Qi, J.M.; Xu, J.T.; Niu, X.P.; Zhang, Y.J.; Tao, A.F.; Zhang, L.W.; Fang, P.P.; Lin, L.H. The CCoAOMT1 gene from jute (Corchorus capsularis L.) is involved in lignin biosynthesis in Arabidopsis thaliana. Gene 2014, 546, 398-402.

20. Zhang, X.R.; Henriques, R.; Lin, S.S.; Niu, Q.W.; Chua, N.H. Agrobacterium-mediated transformation of Arabidopsis thaliana using the floral dip method. Nature Protocol 2006, 2, 1-6.

21. Murashige, T.; Skoog, F. Arevised medium for rapid growth and bioassay with tobacco tissue culture. Physiologia Plantarum 1962, 15, 473-497.

22. Jia, Z.C.; Gou, J.Q.; Sun, Y.M.; Yuan, L.; Tian, Q.; Yang, X.Y.; Pei, Y.; Luo, K.M. Enhanced resistance to fungal pathogens in transgenic Populus tomentosa Carr. by overexpression of an nsLTP-like antimicrobial protein gene from motherwort (Leonurus japonicus). Tree Physiol 2010, 30, 1599-1605.

23. McCown, B.H.; Lloyd, G. Woody Plant Medium (WPM)-a mineral nutrient formulation for microculture for woody plant species. Horticultural Science 1981, $16,453$.

24. Livak, K.J.; Schmittgen, T.D. Analysis of relative gene expression data using real-time quantitative PCR and the $2^{-\Delta \Delta C T}$ method. Methods 2001, 25, 402-408.

25. Fukushima, R.S.; Hatfield, R.D. Comparison of the Acetyl Bromide Spectrophotometric Method with Other Analytical Lignin Methods for Determining Lignin Concentration in Forage Samples. Journal of Agricultural and Food Chemistry 2004, 52, 3713-3720.

26. Lapierre, C.; Pollet, B.; Rolando, C. New Insights Into the Molecular Architecture of Hardwood Lignins by Chemical Degradative Methods. Research on Chemical Intermediates 1995, 21, 397-412.

27. He, B.; Li, Z.G.; Hao, X.J.; He Y.C. A New Technique of Fast Paraffin Sectioning in Plant Tissues. Chinese Bulletin of Botany 2014, 49, 203-208.

28. Raes, J.; Rohde, A.; Christensen, J.; Van de Peer, Y.; Boerjan, W. Genome-Wide Characterization of the Lignification Toolbox in. Plant physiology 2003, 133, 1051-1071. 
29. Chen, L.N.; Guo, X.J.; Yang, H.Q. Cloning and Expression Analysis of CCoAOMT, a Key Gene in Lignin Biosynthesis of Dendrocalamus sinicus. Journal of Plant Genetic Resources 2019, 20, 476-484.

30. Dardick, C.; Callahan, A. Evolution of the fruit endocarp: Molecular mechanisms underlying adaptations in seed protection and dispersal strategies. Frontiers in plant science 2014, 5, 284.

31. Cao, A.J.; Wang, Y.N.; Ge, S.L.; Yang, A.Z.; Xin, Y.J.; Hua, B.G. Changes of enzyme activity during the depositive process of lignin in peach endocarp. Journal of Beijing Agricultural College 2009, 24, 5-8.

32. Zhao, S.G.; Wen, J.; Wang, H.X.; Zhang, Z.H.; Li, X.B. Changes in Lignin Content and Activity of Related Enzymes in the Endocarp During the Walnut Shell Development Period. Horticultural Plant Journal 2016, 3, 141-146.

33. Wang, H.M. Cloning and Functional Characterization of MICCoAOMT1 in Miscanthus. Lutarioriparius. Lanzhou University 2014, Thesis.

34. Jiang, X.H.; She, C.W.; Zhu, Y.H.; Liu, X.M. Cloning and expression analysis of the Lonicera japonica Thunb. chlorogenic acid synthetase gene (LjCCoAOMT1) in rice. Genetics and Molecular Research:GMR 2014, 13, 2166-2176.

35. Guo, D.J.; Chen, F.; Inoue, K.; Blount, J.W.; Dixon, R. Downregulation of Caffeic Acid 3-0-Methyltransferase and Caffeoyl CoA 3-0-Methyltransferase in Transgenic Alfalfa: Impacts on Lignin Structure and Implications for the Biosynthesis of G and S Lignin. The Plant cell 2001, 13, 73-88.

36. Meyermans, H.; Morreel, K.; Catherine, L.; Pollet, B.; Bruyn, A.; Busson, R.; Herdewijn, P.; Devreese, B.; Beeumen, J.; Marita, J.; Ralph, J.; Chen, C.; Burggraeve, B.; Van Montagu, M.; Messens, E.; Boerjan, Wout. Modifications in Lignin and Accumulation of Phenolic Glucosides in Poplar Xylem upon Down-regulation of Caffeoyl-Coenzyme A O-Methyltransferase, an Enzyme Involved in Lignin Biosynthesis. Journal of Biological Chemistry 2000, 275, 36899-36909.

37. Rossitza, A.; Noëlle, F.; Françoise, M.; Brigitte, C.; Marie-Thérèse, T.; Bernard, M.; Bernard, F.; Michel, L. Altered lignin composition in transgenic tobacco expressing 0-methyltransferase sequences in sense and antisense orientation. 
The Plant Journal 2003, 8, 465-477.

38.Ye, Z.H.; Zhong, R.; Morrison, W.H.; Caffeoyl coenzyme A O-methyltransferase and lignin biosynthesis. Phytochemistry 2001, 57, 1177-1185.

39.Schmitt, D.; Pakusch, A.E.; Matern, U. Molecular cloning, induction, and taxonomic distribution of Caffeoyl-CoA 3-0-methyltransferase, an enzyme involved in disease resistance. Journal of Biological Chemistry 1991, 266, 17416-17423.

40. Chapple, C. Molecular-genetic analysis of plant cytochrome P450-dependent monooxygenases. Annual Review of Plant Physiology and Plant Molecular Biology 1998, $49,311-343$.

41. Dai, H.Y.; Han, G.F.; Yan, Y.J.; Zhang, F.; Liu, Z.C.; Li, X.M.; Li, W.R.; Ma, Y.; Li, H.; Liu, Y.X.; Zhang, Z.H.; Transcript Assembly and Quantification by RNA-Seq Reveals Differentially Expressed Genes between Soft-Endocarp and Hard-Endocarp Hawthorns. PLOS ONE 2013, 8, e72910.

42. Ge, H.; Zhang, J.; Zhang, Y.J.; Li, X.; Yin, X.R.; Grierson, D.; Chen, K.S. EjNAC3 transcriptionally regulates chilling-induced lignification of loquat fruit via physical interaction with an atypical CAD-like gene. Journal of Experimental Botany 2017, 68, 5129-5136.

43. Wang, W.Q.; Zhang, J.; Ge, H.; Li, S.J.; Li, X.; Yin, X.R.; Grierson, D.; Chen, K.S. EjMYB8 Transcriptionally Regulates Flesh Lignification in Loquat Fruit. PLOS ONE 2016, 11, e0154399. 